\title{
Factores predictores de mortalidad en pacientes con shock que viven a gran altura
}

\author{
Predicting factors of mortality in patients with shock who live at high altitude
}

Santíago Ruíz-Laos ${ }^{1,2, a *}$, Anibal Díaz-Lazo 1,2,b

Filiación y grado académico

Hospital Regional Docente Clinico Quirurgico Daniel A Carrión, Huancayo, Perú

Universidad Peruana Los Andes, Huancayo, Perú.

Médico Residente de Medicina Intensiva.

Médico Internista-Cardiólogo (Doctor).

D. ORCID iD de Santiago Ruiz

http://orcid.org/0000-0002-5847-2153

(1) ORCID iD de Anibal Diaz

http://orcid.org/0000-0002-9282-9435

Contribución de los autores

SRL: diseño, recolecto datos, proceso los datos, redacto y reviso el contenido del artículo.

ADL: diseño, proceso los datos, redacto y reviso

el contenido del artículo.

Fuentes de financiamiento

No se ha recibido apoyo financiero para

ejecutar la investigación.

Conflictos de interés

ADL: forma parte del equipo editorial de la Revista

Peruana de Ciencias de la Salud, reservándose de participar en el proceso de revisión.

Recibido: 01/10/2018

Arbitrado por pares

Aceptado: 28/02/2019

Citar como

Ruiz S, Díaz-Lazo AV. Factores predictores de mortalidad en pacientes con shock que viven a gran altura. Rev Peru Cienc Salud. 2019; (1): 7-13. doi:https://doi.org/10.37711/rpcs.2019.1.1.4

Correspondencia Santiago Ruiz-Laos Dirección: Chacra Grande Parcela 18 Manzana

E Lote 11. Carabayllo-Lima.

Código postal: 15121

Cel.: 954862003

Email: drgerul@hotmail.com

\section{RESUMEN}

Objetivo. Determinar los factores predictivos de mortalidad en los pacientes con shock que habitan en gran altura a más de 3250 m s.n.m. Métodos. Se realizó un estudio observacional, prospectivo, y analítico, entre diciembre de 2015 y marzo de 2016, en dos Hospitales de Huancayo, Perú. Se incluyeron 46 pacientes con diagnóstico de shock distributivo, hipovolémico o cardiogénico. El instrumento de recolección de datos fue un cuestionario. Para el análisis estadístico se empleó la regresión logística binaria y múltiple. Se consideró significativo $\mathrm{p}<0,05$. Resultados. La edad promedio fue $67+/-18$ años (rango: 9 a 95); 25 (54,3 \%) fueron varones y 21(47,3 \%) mujeres. Fallecieron $32(69,5 \%)$ pacientes. El promedio de edad de fallecidos fue 70,3+/- 9,6 años y de sobrevivientes 50,4 +/- 4,2 años; el tipo de shock más frecuente fue el distributivo y las comorbilidades más comunes fueron insuficiencia respiratoria, hipertensión arterial y diabetes mellitus. Los factores predictivos asociados a mortalidad son: sexo femenino $(85,7 \%$ vs. $14,3 \% ; p=0,02$; edad $\geq$ a 50 años (74,3 \% vs. $25,7 \%$; $p=0,023)$; tener patología previa $(72,7 \%$ vs. $27,3 \% ; p=0,013)$; Saturación Venosa Central de Oxigeno, $\mathrm{SVCO}_{2}$ menor de $70 \%$ (73,1 \% vs. 26,9\%; $p=0,023)$, presentar APACHE II > a 11 puntos y SOFA > a 3 puntos, estuvieron asociados a una alta mortalidad. Conclusión. Los factores de predicción asociados a mortalidad fueron: sexo femenino, edad $>50$ años, tener patología previa, $\mathrm{SVCO}_{2}<70 \%$. El Score APACHE II y SOFA, en altura muestra que a puntajes bajos existe elevada mortalidad.

Palabras clave: choque cardiogénico, Hipovolemia PACHE, Modelos logisticos Perú, Altitud, Estudios prospectivos Conmoción, Hipertensión, Diabetes mellitus, Muerte, Insuficiencia respiratoria, Sobrevivientes, Oxígeno (Fuente: DeCS-Bireme).

\section{ABSTRACT}

Objective. To determine the predictive factors of mortality in patients with shock who live at high altitude at more than 3250 m.a.s.l. Methods. A prospective, observational and descriptive study was conducted between December 2015 and March 2016, at two Hospitals in Huancayo, Peru. We included 46 patients diagnosed with distributive, hypovolemic or cardiogenic shock. The data collection instrument was a questionnaire. For the statistical analysis, binary and multiple logistic regression were used. It was considered significant $\mathrm{p}<0.05$. Results. The average age was $67+/-18$ years (range: 9 to 95); 25 (54.3\%) were male and 21 (47.3\%) female. 32 (69.5\%) patients died. The average age of deaths was $70.3+/-9.6$ years and survivors $50.4+/-4.2$ years; the most frequent type of shock was distributive and the most common comorbidities were respiratory failure, high blood pressure, and diabetes mellitus. The predictive factors associated with mortality were: female sex ( $85.7 \%$ vs. $14.3 \% ; p=0.02$; age $>50$ years $(74.3 \%$ vs. $25.7 \%$; $p=0.023)$; have pathology previous (72.7 \% vs. $27.3 \% ; p=0.013)$; Central Venous Oxygen Saturation, $\mathrm{SVCO}_{2}$ less than $70 \%$ (73.1 \% vs. $\left.26.9 \% ; p=0.023\right)$. Submit APACHE II> at 11 points and SOFA $>$ at 3 points, were associated with high mortality. Conclusions. The prediction factors associated with mortality were: female sex, age $>50$ years, having a previous pathology, $\mathrm{SVCO}_{2}<70 \%$, the APACHE II Score and SOFA, in height shows that at low scores there is high mortality.

Keywords: shock, Cardiogenic, Hypovolemia APACHE, Logistic Models, Peru, Altitude, Prospective Studies Shock, Hypertension, Diabetes Mellitus, Death, Respiratory Insufficiency, Survivors, Oxygen (Source: MeSH-NLM). 


\section{INTRODUCCIÓN}

Existen estudios realizados a nivel del mar, sobre factores de predicción, de mortalidad en general, utilizando las escalas de gravedad como la evaluación de la salud crónica y la fisiología aguda (APACHE por sus siglas en inglés), evaluación de la falla orgánica secuencial (SOFA por sus siglas en ingles), el modelo de predicción de mortalidad (MPM), la calificación de la disfunción orgánica múltiple (DOMS) y otros ${ }^{(1)}$. El Score APACHE es una de las evaluaciones con mayor pronóstico en los últimos años. El APACHE comenzó a utilizarse desde 1981, con 34 variables seleccionadas por un panel de expertos ${ }^{(2,3)}$. El APACHE II se modifica en 1985, con la cual el número de variables se redujo a doce variables fisiológicas ${ }^{(3,4)}$. La evaluación APACHE se divide en tres componentes, la calificación de la fisiología aguda (APS, por sus siglas en inglés), la evaluación de la salud crónica (CHE, por sus siglas en inglés), y la edad; es empleada con mayor frecuencia en diferentes partes del mundo ${ }^{(5,6,7)}$.

Wong et al. ${ }^{(8)}$ realizó un estudio con APACHE II en dos unidades de cuidados intensivos del Canadá ubicada a nivel del mar donde encontró una mortalidad predicha del $24,7 \%$, y observada de $24,8 \%$. Castañeda-Morales et al. ${ }^{(1)}$ en el estudio realizado con 546 pacientes hospitalizados en terapia intensiva en el hospital General de México situado a 2,240 m s.n.m. según APACHE II reportó que la mortalidad predicha fue de $21,1 \%$ y la observada de $40,8 \%$.

Markargf et al. ${ }^{(9)}$ estudiando la precisión de la valoración de modelos predictivos de mortalidad hospitalaria encontró similitud entre lo predicho y hallado según el APACHE II; lo que demuestra un buen grado de calibración y discriminación para este score en altitudes bajas. Según Zimmerman et al. ${ }^{(10)}$, reportan en su estudio realizado en 104 unidades de cuidados intensivos en diversos hospitales de los Estado Unidos de Norteamerica que la precisión de los modelos predictivos de mortalidad es dinámica y debería reevaluarse periódicamente; por lo tanto los resultados encontrados a un nivel de altitud no se pueden generalizar, por lo que se hace necesario hacer estudios en poblaciones que habitan en altura. En nuestro país, hay poca o nula información de estudios realizados en unidades de terapia intensiva ubicadas a gran altitud. Por lo que se diseñó la investigación con el objetivo de determinar los factores predictivos de mortalidad en los pacientes con shock que habitan a gran altura, a más de 3250 m s.n.m.

\section{MÉTODOS}

\section{Tipo y diseño del estudio}

El estudio fue de tipo observacional, prospectivo, y analítico, se realizó entre diciembre de 2015 y marzo de 2016, en la Unidad de Cuidados Intensivos (UCI) del Hospital Regional Docente Clínico Quirúrgico Daniel Alcides Carrión y Hospital Nacional Ramiro Priale Priale de la Ciudad de Huancayo, Perú, ubicado a 3,250 m s.n.m. La población fue de 105 pacientes y la muestra de 46 pacientes hospitalizados. La muestra fue seleccionada de acuerdo a criterios de inclusión y exclusión. Los criterios de inclusión fueron: pacientes con shock distributivo, hipovolémico o cardiogénico; mientras que los de exclusión: pacientes que no presentaron diagnóstico de shock.

\section{Procedimientos de recolección de datos}

Los datos fueron recolectados en un cuestionario con 23 variables. Se calcularon el score APACHE II ${ }^{(3)}$ y SOFA ${ }^{(11)}$, tal como lo establecieron estudios previos. Los puntajes APACHE Y SOFA fueron calculados dentro de las 24 horas posteriores a su ingreso a $\mathrm{UCl}$ y en aquellos pacientes que estuvieron en la $\mathrm{UCl}$ y presentaron Shock. La evaluación se realizó 24 horas después de hecho el diagnóstico.

\section{Análisis de datos}

Para el análisis estadístico se utilizó el $\mathrm{X}^{2}$ para las variables categóricas, $t$ student para las variables continuas y para determinar la predicción de mortalidad se empleó la regresión logística binaria y múltiple. Se usó el paquete estadístico SPSS v20, para el procesamiento de datos. Se consideró significativo $\mathrm{p}<0,05$.

\section{Aspectos éticos}

Se tuvo la aprobación del comité de ética del Hospital Regional Docente Clínico Quirúrgico Daniel Alcides Carrión Huancayo.

\section{RESULTADOS}

Se incluyeron 46 (100\%) pacientes, la edad promedio fue de $67+/-18$ años (rango: 9 a 95); varones 25 $(54,3 \%)$ y mujeres 21 (47,3\%). Fallecieron $32(69,5 \%)$ pacientes y sobrevivieron 14 (30,5\%). La muestra es heterogénea, corresponde a pacientes de diversas edades, 39 (84,8\%) pacientes son mayores de 50 y 7 $(15,2 \%)$ están por debajo de los 50 años $(p=0.023)$. En la figura 1, se muestra las patologías previas más comunes que estuvieron asociadas a shock, encontrándose en primer lugar a las infecciones 


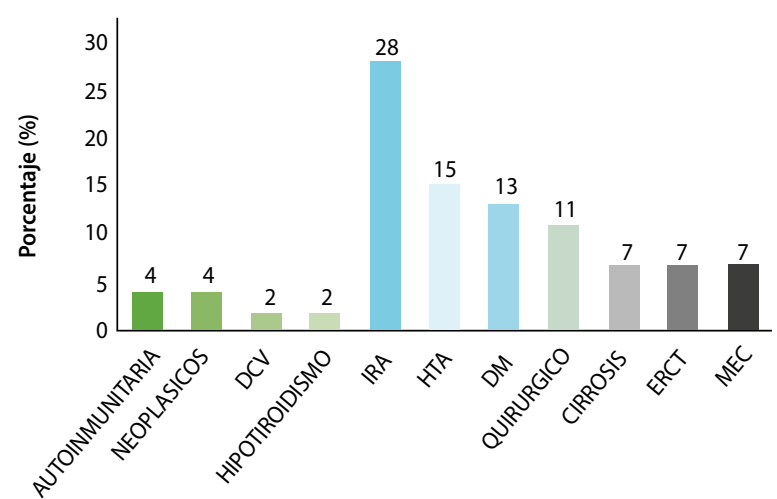

Figura 1. Frecuencias de patologias previas en pacientes con shock

respiratorias (28\%), seguido por la hipertensión arterial (15\%), en tercer lugar se encontró a Diabetes Mellitus (13\%), en cuarto lugar a las intervenciones quirúrgicas (11\%), seguida por la cirrosis, enfermedad renal crónica y las meningoencefalitis con (7\%); cada uno respectivamente.

En la tabla 1, se muestra los factores predictivos asociados a mortalidad las cuales fueron: sexo femenino $(85,7 \%$ vs. $14,3 \% ; p=0,02)$; edad > a 50 años (74,3\% vs. $25,7 \%$; $p=0,023)$; tener patología previa $(72,7 \%$ vs. $27,3 \% ; p=0,013)$; saturación venosa central de oxígeno, $\mathrm{SVCO}_{2}$ menor de $70 \%$ (73,1 \% vs. 26,9\%; $\mathrm{p}=0,023)$. Presentar APACHE II > a 11 puntos y SOFA > a 3 puntos, quienes estuvieron asociados al $100 \%$ de mortalidad.

En la tabla 2, se muestra que entre los 16 a 20 puntos obtenidos al aplicar el score APACHE II, la

Tabla 2. Porcentaje de predicción de mortalidad de pacientes con shock que habitan a gran altura, según score APACHE II

\begin{tabular}{lccc}
\hline $\begin{array}{c}\text { Puntaje de } \\
\text { Score APACHE } \\
\text { II }\end{array}$ & $\begin{array}{c}\text { Total casos } \\
\text { según Score } \\
\text { APACHE II } \\
\mathbf{n}=\mathbf{4 6}\end{array}$ & $\begin{array}{c}\text { Total de Casos } \\
\text { Fallecidos } \\
\text { según Score } \\
\text { APACHE II } \\
\mathbf{n = 3 2}\end{array}$ & $\begin{array}{c}\text { \% de } \\
\text { Predicción de } \\
\text { Mortalidad a } \\
\text { Gran Altura }\end{array}$ \\
\hline 0 a 5 & 0 & 0 & 0,0 \\
6 a 11 & 0 & 0 & 0,0 \\
11 a 15 & 9 & 3 & 33,3 \\
16 a 20 & 10 & 8 & 80,0 \\
21 a 25 & 14 & 10 & 71,4 \\
26 a 30 & 7 & 6 & 85,7 \\
> 31 puntos & 6 & 5 & 83,3 \\
\hline
\end{tabular}

Tabla 1. Factores predictivos de mortalidad en pacientes con shock que habitan a $3250 \mathrm{~m}$ s.n.m

\begin{tabular}{lccccc}
\hline \multirow{2}{*}{$\begin{array}{l}\text { Factores predictivos de } \\
\text { mortalidad }\end{array}$} & \multicolumn{2}{c}{$\begin{array}{c}\text { Fallecidos } \\
\mathbf{n}=\mathbf{3 2}\end{array}$} & $\begin{array}{c}\text { Sobrevivientes } \\
\mathbf{n = 1 4}\end{array}$ & $\boldsymbol{p}<\mathbf{0 , 0 5}$ \\
\cline { 2 - 5 } & $\mathbf{f i}$ & $\%$ & $\mathbf{f i}$ & $\%$ & \\
\hline $\begin{array}{l}\text { Sexo } \\
\quad \text { Mujeres }\end{array}$ & 18 & 85,7 & 3 & 14,3 & $p=0,020$ \\
$\quad$ Varones & 14 & 56,0 & 11 & 44,0 & \\
$\quad$ Edad & & & & & \\
$\quad<50$ años & 3 & 42,9 & 4 & 57,1 & $p=0,023$ \\
$\quad>50$ años & 29 & 74,3 & 10 & 25,7 & \\
Poseer Patología Previa & & & & & \\
$\quad$ Sín & 31 & 72,7 & 12 & 27,3 & $p=0,013$ \\
$\quad$ No & 1 & 0 & 2 & 100 & \\
$\quad$ Saturación Venosa Central & & & & & \\
de Oxigeno (SvcO $)$ & & & & & \\
$\quad<70 \%$ & 30 & 73,1 & 11 & 26,9 & \\
$\quad>70 \%$ & 2 & 40,0 & 3 & 60,0 & $p=0,023$ \\
\hline
\end{tabular}

predicción de la mortalidad es muy alta (80\%), en los pacientes con shock que viven a más de 3250 m s.n.m.

En la tabla 3, se muestra que entre los 16 a 20 puntos según el score APACHE II la mortalidad es muy alta (80 \%) en pacientes con shock que viven a gran altitud (3250 m s.n.m.) en comparación a quienes habitan a nivel del mar (16,4\%); esta diferencia estadísticamente es significativa, además existe una relación de 21 a 1 entre los fallecidos versus los vivos.

En la tabla 4, se muestra que entre los 7 a 9 puntos, según el score SOFA, la mortalidad es alta $(68,2 \%)$, y esta se incrementa aún más entre 10 a 12 puntos $(87,5 \%)$ en pacientes con shock que viven a gran altitud (3250 m s.n.m.).

Tabla 3. Comparación de predicción de mortalidad del score APACHE II en pacientes a nivel del mar y que habitan a gran altura

\begin{tabular}{|c|c|c|c|c|}
\hline \multirow{2}{*}{$\begin{array}{l}\text { Puntaje de } \\
\text { Score } \\
\text { APACHE II* }\end{array}$} & \multicolumn{4}{|c|}{ Porcentaje de Predicción de mortalidad } \\
\hline & $\begin{array}{c}\text { A Nivel del } \\
\text { Mar* } \\
(\%)\end{array}$ & $\begin{array}{c}\text { A Gran } \\
\text { Altura** } \\
(\%)\end{array}$ & $\begin{array}{c}\text { OR } \\
\text { (IC } 95 \%)\end{array}$ & Valor - p \\
\hline 0 a 5 & 2,3 & 0,0 & & \\
\hline 6 a 11 & 4,3 & 0,0 & & \\
\hline 11 a 15 & 8,6 & 33,3 & $4,9(2,2-11,1)$ & $p<0,0001$ \\
\hline 16 a 20 & 16,4 & 80,0 & $\begin{array}{c}21.0 \\
(10,1-43,3)\end{array}$ & $\mathrm{p}<0,0001$ \\
\hline 21 a 25 & 28,2 & 71,4 & $6,2(3,4-11,6)$ & $p<0,0001$ \\
\hline 26 a 30 & 56,4 & 85,7 & $4,8(2,4-9,6)$ & $p<0,0001$ \\
\hline$>31$ & 70 & 83,3 & $2,09(1,0-4,1)$ & $p=0,0320$ \\
\hline
\end{tabular}


Tabla 4. Porcentaje de predicción de mortalidad de pacientes con shock que habitan a gran altura, según score SOFA

\begin{tabular}{lccc}
\hline $\begin{array}{c}\text { Puntaje de } \\
\text { Score SOFA }\end{array}$ & $\begin{array}{c}\text { Total casos } \\
\text { según } \\
\text { Score SOFA } \\
\mathbf{n = 4 6}\end{array}$ & $\begin{array}{c}\text { Total de Casos } \\
\text { Fallecidos } \\
\text { según Score } \\
\text { SOFA } \\
\mathbf{n = 3 2}\end{array}$ & $\begin{array}{c}\% \text { de } \\
\text { Predicción de } \\
\text { Mortalidad a } \\
\text { Gran Altura }\end{array}$ \\
\hline 0 a 6 & 16 & 8 & 50,0 \\
7 a 9 & 22 & 15 & 68,2 \\
10 a 12 & 8 & 7 & 87,5 \\
13 a 14 & 0 & 0 & 0,0 \\
15 & 0 & 0 & 0,0 \\
16 a 24 & 0 & 0 & 0,0 \\
\hline
\end{tabular}

En la tabla 5, se muestra que entre los 7 a 9 puntos, según el score SOFA, la mortalidad se incrementa en porcentaje significativo $(68,2 \%)$ en pacientes con shock que viven a gran altitud (3250 m s.n.m.) en comparación a quienes habitan a nivel del mar donde el porcentaje de predicción de mortalidad es bajo (15 a $20 \%$ ). En altura a puntajes bajos, según SOFA, el porcentaje de predicción de mortalidad es alta en comparación a quienes habitan a nivel del mar.

\section{DISCUSIÓN}

En muchas unidades de terapia intensiva se han hechos estudios para evaluar escalas pronosticas, siendo una de las más utilizadas el score APACHE II, para cuantificar la gravedad de los pacientes que ingresan; sin embargo, no siempre es posible determinar con exactitud y objetivamente su valor pronostico ${ }^{(12,13)}$. Por otro lado, diversos estudios realizados en diferentes países reportaron una mortalidad que varía entre $17 \%$ a $36 \%{ }^{(13)}$. También existe los criterios de evaluación secuencial de falla orgánica (SOFA) que es útil para aplicar en los pacientes con shock séptico que continúa siendo una causa importante de ingreso a cuidados intensivos y de muerte ${ }^{(14)}$.
En un estudio realizado por Penott-Gutierrez et al. (15) reporta una mortalidad del $25,0 \%$, con una muestra similar a la de nuestro estudio, donde todos los pacientes presentaban shock séptico. La mortalidad encontrada en nuestra serie fue 69,6 \% (32) de los pacientes; un porcentaje muy alto en relación a otros estudios. En nuestro estudio, se encontró que la edad promedio de los fallecidos fue de 70,3 +/- 9,6 años, siendo muy superior a la encontrada en la serie reportada por Penott-Gutierréz et al. ${ }^{(15)}$ que fue de $51,6+-10,9$ años, mientras que el promedio de edad de los sobrevivientes entre ambos estudios no hubo una gran diferencia (45,4 +-11,3 vs 50,4+- 4,2 años); por lo que podemos inferir que en altura una edad avanzada estuvo asociado como factor predictor de mortalidad.

El 95,7\% (44) de los pacientes estudiados, presento una patología previa de fondo, que no fue necesariamente motivo de ingreso, algunas de las enfermedades que se presentaron son consecuencia relativa, de una mejora de la calidad de vida en la población; sin embargo existe un incremento de enfermedades crónicas degenerativas no infecciosas que se encuentran en aumento; como son: la diabetes y la hipertensión arterial; por otro lado se evidencia la existencia de saneamientos ambientales inadecuados; como son: el consumo de alimentos cocidos con fuego de leña, o el laborar en centros mineros, que con el transcurso del tiempo producen diversas enfermedades crónicas como la fibrosis pulmonar, que en nuestro medio aun es frecuente.

Es importante el estudio de la enfermedad previa porque esta está directamente relacionada con la calidad de vida y con el pronóstico del paciente por la probable falla orgánica o multiorgánica con que se presenta desde el inicio el paciente. En nuestra serie se evidenció que el poseer patología previa

Tabla 5. Comparación de predicción de mortalidad del score SOFA en pacientes a nivel del mar y que habitan a gran altura

\begin{tabular}{lccc}
\hline Puntaje de Score SOFA* & $\begin{array}{c}\text { \% de } \\
\text { Predicción de Mortalidad a } \\
\text { Nivel del Mar* }\end{array}$ & $\begin{array}{c}\text { Predicción de Mortalidad a } \\
\text { Gran Altura }\end{array}$ & \begin{tabular}{c} 
OR (IC 95\%) \\
Valor - p \\
\hline a 6
\end{tabular} \\
7 a 9 & $15-20$ & 50,0 & $9,0(4,2-19,2)$ \\
10 a 12 & $40-50$ & 68,2 & $8,5(4,4-16,2)$ \\
13 a 14 & $50-60$ & 87,5 & $6,6(3,3-13,5)$ \\
15 & $>80$ & & $p<0,0001$ \\
16 a 24 & $>90$ & & \\
\hline
\end{tabular}


está relacionado a una alta predicción de mortalidad $(72,7 \% ; p<0,05)$, estadísticamente significativa.

En el Perú y en la mayoría de los países de Latino América, las enfermedades del tracto respiratorio se encuentran dentro de las diez primeras causas de morbilidad. En el estudio se encontró que dicha patología fue la primera causa de motivo de ingreso al hospital (28,0\%), seguido por la hipertensión arterial (15\%) y diabetes mellitus $2(13 \%)$. Estas enfermedades crónicas degenerativas no infecciosas en los últimos años se han incrementado en la población de nuestro país ${ }^{(16)}$. Otro grupo de enfermedades está referido a diversas patologías infecciosas, complicaciones quirúrgicas, neoplásicas y complicaciones de la hipertensión arterial, como resultados del inadecuado cuidado básico de la salud o de la prevención de la misma. En contraste con nuestro estudio, en la investigación realizada por Sendra et al, (17) se reporta que la primera causa de mortalidad fueron: las enfermedades circulatorias, seguida del infarto de miocardio agudo. Sin embargo en el estudio de Regueira ${ }^{(18)}$, encuentra que la principal causa fue el shock séptico, seguido del síndrome de disfunción multiorgánica.

En nuestra casuística se establece que el sexo femenino es un factor predictor de la mortalidad, las mujeres fallecieron más que los varones $(85,7 \%$ vs $56 \% ; p=0,020$ ). En el estudio realizado por Sendra et al. ${ }^{(17)}$, se hace referencia a que el sexo es un factor asociado a la mortalidad, presentándose el doble de mortalidad en las mujeres en comparación a los varones; pero así mismo menciona que el riesgo de mortalidad tiende a disminuir cuando se realiza el ajuste con el resto de las variables consideradas. Sin embargo en el estudio de Penot-Gutierrez et al. ${ }^{(15)}$ realizado en Mérida, una ciudad Venezolana, ubicada a $1600 \mathrm{~m}$ s.n.m. establece que no encontró diferencia estadísticamente significativa entre la variable sexo y la mortalidad. En nuestra serie realizada a 3250 m.s.n.m. encontramos que existe una relación en la mortalidad entre el sexo femenino y masculino de 1.5.

Al analizar la edad como un factor predictor de mortalidad, se obtuvo que conforme aumentaba la edad, se incrementaba la mortalidad; tal como se hizo evidente en los pacientes mayores de 50 años de edad, alcanzando un porcentaje elevado de mortalidad de $74,3 \%$ fallecidos versus el $25,7 \%$ de los sobrevivientes. En el estudio de Labelle et al. ${ }^{(19)}$ se encuentra que la edad es un factor significativo de mortalidad con relación a la supervivencia; así mismo en el estudio de Penott-Gutierrez et al. ${ }^{(15)}$ hace referencia a que conforme aumenta la edad incrementa la mortalidad.

En nuestro estudio se encontró una relación significativa entre la mortalidad y la saturación venosa central $\left(\mathrm{SvCO}_{2}\right)$ observándose que a menor saturación, existe mayor mortalidad, el $73,1 \%$ de los pacientes fallecieron cuyo valores de $\mathrm{SvcO}_{2}$ fueron menores o igual a $70 \%$, $(p<0,023)$. Penott-Gutierrez et al. ${ }^{(15)}$ en su serie, también encontró relación con la saturación venosa central. Sin embargo en los datos obtenidos por el ultimo investigador, se muestra que en el grupo de sobrevivientes los valores de cohorte fueron de $70,6+/-4,1$ y en los fallecidos fue de $65,9+/-7,5$ valores por debajo a lo encontrado en nuestra serie. En investigaciones previas se menciona que el hecho de presentar $\mathrm{SvCO}_{2}$ bajas, es un factor determinante en la condición y pronóstico del paciente ${ }^{(20)}$. Además se reporta que una $\mathrm{SvcO}_{2}<60$, presentan mayores tasas de mortalidad ${ }^{(15)}$. Por lo tanto consideramos que una $\mathrm{SvCO}_{2}<70$, es un factor predictor de mortalidad en pacientes con shock, por la alta mortalidad encontrada en nuestro trabajo realizado en la altura.

Como se aprecia en los resultados, 41 (89,1\%) pacientes, presenta valores de saturación venosa central de oxigeno $<70 \%$; lo que podría pensarse que dicha población se encuentra con un incremento en la demanda de oxígeno y un descenso en la disponibilidad, lo que teóricamente indicaría que la disponibilidad de oxigeno es menor a la demanda y probablemente el inicio de la acidosis láctica con el deterioro orgánico respectivo. Asimismo, solo 5 $(10,9 \%)$ pacientes de la muestra presentaron una saturación venosa central con valores por encima del $70 \%$, en donde probablemente la disponibilidad es mayor a la demanda. En el estudio de Dueck et al. ${ }^{(20)}$ se demostró que aquellos pacientes que no sobrevivieron, presentaron los valores más bajos de la saturación venosa central. Según Briceño (21), el desarrollo de la sepsis y el shock séptico involucra varios cambios patológicos, incluyendo hipoxia tisular global como resultado de las anomalías circulatorias.

Según la puntuación obtenida aplicando el score APACHE, desde valores de 11 puntos en los pacientes observados ya existe una alta predicción de mortalidad. Asimismo, se tiene la información que en los pacientes a nivel del mar con más de 31 puntos presentan una predicción de mortalidad de más del $70 \%$, mientras que en el presente trabajo se observó que a partir de 16 puntos del Score APACHE, los pacientes presentaron una predicción de mortalidad 
mayor del $80 \%$; por lo que la predicción de mortalidad es cinco veces mayor en la altura que a nivel del mar, según el score APACHE.

En el estudio de Landa et al. (2010), realizado a nivel del mar, destaca la puntuación del APACHE II inicial; como promedio tuvo en 23,4 puntos para los fallecidos y 13,7 puntos en los vivos. También los valores del APACHE II inicial, estuvieron relacionados con una mayor mortalidad (22). Sin embargo, en el estudio realizado en el Hospital Universitario de Maracaibo Venezuela, situado a 1600 m s.n.m. concluyó que el score APACHE II, en el grupo de sobrevivientes, el punto de cohorte fue de 20,4 +/-5, 1 puntos y para los fallecidos fue de $18,3+/-4,0$ puntos sin diferencias significativas ${ }^{(15)}$.

Blas et al. ${ }^{(23)}$ en Durango-México ubicado a 1884 msnm aplicando el score APACHE II a pacientes hospitalizados en cuidados intensivos, encontró una mortalidad predicha de $29,8 \%$ y observada de $32,7 \%$. Castañeda-Morales et al. ${ }^{(1)}$ a 2240 m s.n.m reportó que la mortalidad predicha fue de $21,1 \%$ y la observada de 40,8 \%. Según Aguirre-Serrato et al. estudiando 95 casos que ingresaron a la Unidad de Terapia Intensiva del Hospital Español de México reporta una mortalidad predicha de $28,6 \%$ y observada de $20 \%$, según score APACHE II. Khwannimit et al. (25), en su estudio menciona que el score APACHE II mostró pobre calibración en predecir la mortalidad en pacientes hospitalizados en $\mathrm{UCl}$.

En el estudio realizado por Lange et al. (2006) se demostró la utilidad del score APACHE, ya que describió que la probabilidad de mortalidad estaba directamente relacionado con altos puntajes del score APACHE. De acuerdo a varios estudios realizados, los valores de APACHE II han sido una herramienta que se ha utilizado para predecir la mortalidad en pacientes severamente comprometidos, independientemente del lugar de hospitalización ${ }^{(12)}$. Si comparamos los estudios de Landa et al. ${ }^{(22)}$ y de Penott-Gutierrez et al ${ }^{(15)}$, con lo establecido por Knauss et al. en el año 1985, se observa que altos puntajes encontrados, están relacionado con una elevada mortalidad (3). Además, Sadaka et al. ${ }^{(26)}$ refiere que el score APACHE Il es un fuerte predictor de mortalidad hospitalaria en pacientes sépticos con buena discriminación y poca calibración.

Asi mismo Sittig et al. (27) plantea en su trabajo de investigación que las condiciones en una misma zona geográfica entre los pacientes son similares; sin embargo varían de zona en zona y por las condiciones peculiares de la altura el nivel de puntuación del APACHE, debería de ser menor en comparación a ciudades de menor altura ${ }^{(27)}$. En nuestro estudio se encontró según lo planteado por Sittig et al. (26) bajos puntajes con una alta mortalidad; por lo que consideramos un factor predictivo de mortalidad importante y un aporte en el manejo de nuestros pacientes en altura.

Por otro lado, el estudio de la escala del SOFA es importante porque valora el estado general del pacientey sobre todo al inicio, cuando esta es de menos de tres puntos; sin embargo una valoración mayor de los 3 puntos indicaría un daño orgánico presente, por lo que la mortalidad se aumenta considerablemente. En este sentido, el SOFA constituye la mejor de las escalas predictivas; sobre todo si se evalúa diariamente el progreso o la mejoría del Síndrome de Disfunción Multi Orgánica (SDMO) ${ }^{(13)}$.

En el estudio realizado, se encontró que el intervalo de la puntuación del Score SOFA, va de 0 a 12 puntos; además se evidenció la alta probabilidad de mortalidad (68,2\%), en pacientes con puntaje de 7 a 9 puntos, cuatro veces mayor que las de nivel del mar, cuya predicción de mortalidad es del $15 \%$ al $20 \%$ con el mismo puntaje. En la investigación de Penott-Gutierrez et al. ${ }^{(15)}$ se concluye que el Score SOFA, no fue significativo, puesto que en su muestra, obtuvieron prácticamente el mismo puntaje entre los sobrevivientes y fallecidos $(8,3+/-3,9$ vs 8,0 $+/-5,8$ ); también menciona la baja probabilidad de mortalidad, a diferencia de nuestro estudio donde se encontró que a bajos puntajes del score SOFA existe una alta mortalidad, constituyéndose como un factor predictivo desfavorable.

Una de las limitaciones del estudio es la poca cantidad de pacientes incluidos en el estudio; a pesar de ello un aporte importante del estudio es que a bajo puntajes del score APACHE II y SOFA se observa una elevada mortalidad; lo que está relacionado con la edad avanzada, la condición muy grave y las comorbilidades que presentan los pacientes cuando ingresan a la UCl.

Se concluye que los factores de predicción asociados a mortalidad fueron: sexo femenino, edad $>$ a 50 años, tener patología previa y $\mathrm{SVCO}_{2}<$ a $70 \%$. Los niveles de Score APACHE y SOFA, encontrados en la altura no se relacionan con la predicción de mortalidad a nivel del mar, porque a puntajes bajos de ambos score existe una alta mortalidad en la altura. 


\section{REFERENCIAS BIBLIOGRÁFICAS}

1. Castañeda-Morales VM, Sanchez-Velasquez LD, Jimenez-Garduño AM. Calibración y discriminación del APACHE II y del APACHE IV. Rev Mex Med Crit y Ter Int. 2013; 27(1): 8-14

2. Knaus WA, Zimmerman JE, Wagner DP, Draper EA, Lawrence DE. APACHE-Acute Physiology And Chronic Health Evaluation: A physiologically based classifi cation system. Crit Care Med. 1981; 9: 591-7.

3. Knaus WA, Draper EA, Wagner DP, Zimmerman JE, Birnbaum ML, Cullen DJ, et al. Evaluating outcome from intensive care: A preliminary multihospital comparison. Crit Care Med .1982; 10(8): 491-6.

4. Knaus WA, Draper EA, Wagner DP, Zimmerman JE. APACHE II: A severity of disease classification system. Crit Care Med. 1985; 13: 818-29.

5. Knaus WA, Wagner DP, Draper EA, Zimmerman JE, Bergner M, Bastos PG, et al. The APACHE III prognostic system. Risk prediction of hospital mortality for critically ill hospitalized adults. Chest. 1991; 100: 1619-36.

6. Glance LG, Osler TM, Dick A. Rating the quality of intensive care units: Is it a function of the intensive care unit scoring system? Crit Care Med. 2002; 30: 1976-82.

7. Rowan KM, Kerr JH, Major E, McPherson K, Short A, Vessey MP. Intensive Care Society's APACHE II study in Britain and Ireland--II: Outcome comparisons of intensive care units after adjustment for case mix by the American APACHE II method. BMJ. 1993; 307: 977-81.

8. Wong DT, Crofts SL, Gomez M, McGuire GP, Byrick RJ. Evaluation of predictive ability of APACHE II system and hospital outcome in Canadian intensive care unit patients. Crit Care Med .1995; 23: 1177-83.

9. Markgraf R, Deutschinoff G, Pientka L, Scholten T. Comparison of Acute Physiology And Chronic Health Evaluations II and III and Simplifi ed Acute Physiology Score II: A prospective cohort study evaluating these methods to predict outcome in a German interdisciplinary intensive care unit. Crit Care Med. 2000; 28(1): 26-33. doi: 10.1097/00003246-200001000-00005

10. Zimmerman JE, Kramer AA, McNair DS, Malila FM. Acute Physiology and Chronic Health Evaluation (APACHE) IV: Hospital mortality assessment for today's critically ill patients. Crit Care Med. 2006; 34: 1297-310. DOI:10.1097/01.CCM.0000215112.84523.F0

11. Vincent JL, Moreno R, Takala J, Willats S, De Mendoca A, Bruining $\mathrm{H}$. The SOFA (sepsis related organ failure assessment) score to describe organ dysfunction/ failure on behalf of the working group on sepsis relatedproblems of the European Society of Intensive Care Medicine. Intensive Care Med. 1996 jul; 22(7): 707-10

12. Lange J, Reyes-Prieto $M$, Sosa M, Ojeda J. Utilidad del score APACHE en terapia intensiva, Corrientes: Universidad Nacional del Nordeste; 2006.
13. Gien-Lopez JA, Salazar- Escalante D, Lopez R, Ramirez JJ. Valor predictivo de la escala APACHE II sobre la mortalidad en una unidad de cuidados intensivos de adultos en la ciudad de Merida Yucatan. Rev Mex Med Crit y Ter Int. 2006; 20(1): 30-40.

14. Zhang W, Danzeng Q, Feng X, Cao X, Chen W, Kang Y. Sequential organ failure assessment predicts outcomes of pulse indicator contour continuous cardiac-output directed goal teraphy: A prospective study. Medicine (Baltimore). 2017; 96(39): e8111.

15. Penott-Gutierrez O, Reyna-Villasmil E. Saturación venosa central de oxígeno inicial como predictor de mortalidad en pacientes con sepsis. MedULA. 2013; 22 (1): 15-20.

16. Ministerio de Salud.Boletin epidemiológico, quinquenio 2011-2015. Lima: MINSA; 2015.

17. Sendra JM, Sarria-Santamera A, Iñigo J, Regidor E. Factores asociados a la mortalidad intrahospitalaria del infarto de miocardio. Resultados de un estudio observacional. Med Clin. 2005; 125(17): 641-646.

18. Regueira T. Manipulacion y transporte de oxígeno en la sepsis, Pontificia Universidad Católica de Chile. Rev Med Chile. 2010; 138: 233-242.

19. Labelle A, Juang P, Reichley R, Micek S. Crit Care Med. Mortalidad Hospitalaria en pacientes con shock séptico. Crit Care Med. Missouri, USA. 2012; 40: 2016-2021.

20. Dueck M, Klimek M, Appenrodt S, Weigand C, Boerner $U$. Trends but not individual values of central venous oxygen saturation agree with mixed venous oxygen saturation during varying hemodynamic conditions. Anesthesiology. 2005; 103(2): 249-257.

21. Briceño I. Sepsis: etiología, manifestaciones clínicas y diagnóstico. Medicrit. 2005; 2(9): 203-213.

22. Landa AL, Rubiera R, Sordo R. Valoración del APACHE II inicial como predictor de mortalidad en pacientes ventilados. Rev Cub Med Int Emerg. 2010; 9(3): 1771-87.

23. Blas-Macedo J, Juárez-Olvera A. Mortalidad en la unidad de cuidados intensivos: Evaluación con una escala pronóstica. Rev Mex Med Crit y Ter Int. 2001;15(2): 41-4.

24. Aguirre-Serrato CA, Cerón-Díaz UW, Sierra-Unzueta A. Comparación del rendimiento de 2 modelos predictivos de mortalidad: SAPS 3 versus APACHE II, en una unidad de terapia intensiva mexicana. Rev Mex Med Crit y Ter Int 2007: 21(3):119-24.

25. Khwannimit B , Geater A. A comparison of APACHE II and SAPS II scoring Systems in predicting hospital mortality in Thai adult intensive care units. J Med Assoc Thai. 2007; 90(4): 643-52.

26. Sadaka F, EthmaneAbouEIMaali Ch, Cytron MA, Fowler K, Javaux VM, O'Brien J. Predicting Mortality of patients with sepsis: A comparison of APACHE II and APACHE III Scoring Systems. J Clin Med Res. 2017; 9(11): 907-910. doi: 10.14740/jocmr3083w

27. Sittig DF, Gardner RM, Menlove RL, Clemmer TP. APACHE II, modification for use at high altitude. Crit Care Med. 1987 Sep; 15(9): 890-1. 